\title{
Strain Coupling, Microstructure Dynamics and Acoustic Mode Softening in Germanium Telluride
}

\author{
D. Yang ${ }^{1,2}$ T. Chatterji. ${ }^{3}$ J. A. Schiemer, ${ }^{1}$ M. A. Carpenter ${ }^{1}$ \\ ${ }^{1}$ Department of Earth Sciences, University of Cambridge, Cambridge CB2 3EQ, United Kingdom \\ ${ }^{2}$ School of Materials Science and Technology, China University of Geosciences, Beijing 100083, People's Republic of
}

China

${ }^{3}$ Institut Laue-Langevin, B.P. 156, 38042 Grenoble Cedex 9, France

\begin{abstract}
GeTe is a material of intense topical interest due to its potential in the context of phase-change and nanowire memory devices, as a base for thermoelectric materials and as a ferroelectric. The combination of a soft optic mode and a Peierls distortion contributes large strains at the cubic - rhombohedral phase transition near $625 \mathrm{~K}$ and the role of these has been investigated through their influence on elastic and anelastic properties by resonant ultrasound spectroscopy. The underlying physics is revealed by softening of the elastic constants by $\sim 30-45 \%$, due to strong coupling of shear and volume strains with the driving order parameter and consistent with an improper ferroelastic transition which is weakly first order. The magnitude of the softening is permissive of the transition mechanism involving a significant order/disorder component. A Debye loss peak in the vicinity of $180 \mathrm{~K}$ is attributed to freezing of the motion of ferroelastic twin walls and the activation energy of $\sim 0.07 \mathrm{eV}$ is attributed to control by switching of the configuration of long and short Ge-Te bonds in the first coordination sphere around Ge. Precursor softening as the transition is approached from above can be described with a Vogel-Fulcher expression with a similar activation energy, which is attributed to coupling of acoustic modes with an unseen central mode that arises from dynamical clusters with local ordering of the Peierls distortion. The strain relaxation and ferroelastic behaviour of GeTe depend on both displacive and order/disorder effects but the dynamics of switching will be determined by changes in the configuration of distorted $\mathrm{GeTe}_{6}$ octahedra, with a rather small activation energy barrier.
\end{abstract}

PACS numbers: 62.40.+i, 64.60.Ej, 77.84.Bw

\section{INTRODUCTION}

GeTe is a remarkable material in several topical contexts, including as an end-member phase for crystal-to-amorphous phase change memory [1-4], for nanowire memory devices [5], as a base for thermoelectric materials [6.7] and as a ferroelectric at room temperature which retains "a reversible, size dependent polar-nonpolar transition in nanocrystal ensembles" [8]. Close interest arises because of the particular combination of structure and electronic properties which can give fast switching and stable storage. These in turn depend on high vacancy substitution for Ge $[9,10]$ and a Peierls-type distortion of the Ge-Te coordination which persists even above the melting point $[3,11-14]$. The Peierls distortion can also be described as a second order Jahn-Teller distortion of the first coordination sphere of Te around $\mathrm{Ge}$, due to the formation of Te lone pairs [15].

In addition to the implications for device applications, there has been controversy in relation to the origin of ferroelectric properties that arise at a cubic $(F m 3 m)$ - rhombohedral $(R 3 m)$ phase transition at $\sim 600-700 \mathrm{~K}$ [7,14,16-19] because the electronic instability which gives rise to the Peierls distortion would give the same change in symmetry as a soft optic mode. A classical displacive transition driven by a zone centre optic mode was implied by Raman scattering results, which showed mode softening up to at least 500 $\mathrm{K}$ [18] and would be consistent with the equivalent transition observed at lower temperatures in $\mathrm{Sn}_{\mathrm{x}} \mathrm{Ge}_{1-\mathrm{x}} \mathrm{Te}$ [20] and $\mathrm{Pb}_{\mathrm{x}} \mathrm{Ge}_{1-\mathrm{x}} \mathrm{Te}$ [21]. This displacive mechanism was supported by theory [22], by inelastic neutron scattering data from a powder sample and by computer simulations [23]. However, probes of the local structure have shown that the Peierls distortion persists locally in the structure at all temperatures up to the melting point and, hence, that the transition must involve at least some order/disorder of distorted units [13-15].
Conventional memory devices depend on switching, with characteristic mechanisms that involve movement of twin walls in response to an applied field. In the case of GeTe, the mechanism of ferroelectric switching will depend at a local scale on reversing the topology of the three long and three short bonds of individual distorted $\mathrm{GeTe}_{6}$ octahedra [24]. As well as being ferroelectric, the phase transition in GeTe is improper ferroelastic, however, and it is inevitable that the rhombohedral phase will contain ferroelastic $\left(71 / 109^{\circ}\right)$ twin walls which will also move under an applied electric field, though with different dynamics from $180^{\circ}$ twin walls. This is normal in ferroelectrics such as $\mathrm{BaTiO}_{3}$ and $\mathrm{Pb}(\mathrm{Zr}, \mathrm{Ti}) \mathrm{O}_{3}$ but leads to the expectation that GeTe must also display diverse acoustic properties which have not yet been investigated. The primary objective of the present study was to use measurements of elastic and anelastic properties to reveal the phenomenological richness and underlying physics of a material which, superficially at least, is just a binary compound with the rocksalt structure.

Here we show, firstly, that softening of the shear elastic constants due to classical strain/order parameter coupling is consistent with a predominantly displacive mechanism for the proper ferroelectric/improper ferroelastic transition, but with significant contribution from order/disorder. Secondly, we argue that precursor softening in the stability field of the cubic structure provides indirect evidence of a central relaxational mode which arises from some dynamical microstructure of polar nanoregions or tweed and which couples with acoustic modes. Finally, we suggest that the activation energy of $\sim 0.1 \mathrm{eV}$ extracted from both a peak in the acoustic loss due to freezing of ferroelastic twin walls near $200 \mathrm{~K}$, which is consistent with formalism from the Debye equations [25], and from a Vogel-Fulcher description of the precursor softening is due to motion of $\mathrm{Ge}$ between the two alternative sites of the Peierls 
structure. This is likely to be fundamental to the dynamical response of GeTe to external fields.

\section{STRAIN ANALYSIS}

From the perspectives of strain and elasticity, the weakly first order cubic - rhombohedral transition in GeTe appears to have all the typical features of being improper ferroelastic. A formal strain analysis, based on a Landau expansion with strain/order parameter coupling using lattice parameter data from the literature, is given in the Appendix. Values of the symmetry breaking shear strain, $e_{4}$, and volume strain, $e_{\mathrm{a}}$, are $\sim 3 \%$ and $\sim 1.8 \%$ at room temperature, signifying strain coupling comparable in strength to that which accompanies Jahn-Teller transitions in perovskites such as $\mathrm{LaMnO}_{3}$ [26], but stronger by a factor of $\sim 10$ than accompanies octahedral tilting transitions in perovskites such as $\mathrm{SrZrO}_{3}$ [27]. Variations of these strains with temperature can be represented by the standard solution to a Landau 2-4-6 potential for a weakly first order displacive transition, i.e. with negative fourth order coefficient and a small difference between the transition temperature, $T_{\mathrm{tr}}$, and critical temperature, $T_{\mathrm{c}}$.

\section{EXPERIMENTAL DETAILS}

The elastic and anelastic properties of a single crystal sample cut in the shape of a rectangular parallelepiped $\left(1.977 \times 3.283 \times 4.611 \mathrm{~mm}^{3}, 0.1822 \mathrm{gm}\right.$, but with no particular crystallographic orientation) were measured by resonant ultrasound spectroscopy (RUS). Different pieces of the same boule had previously been used for neutron diffraction [17] and synchrotron X-ray diffraction [15]. Examination of one or the offcuts by electron backscatter diffraction (EBSD) confirmed that it was a single crystal.

The RUS method has been described in detail elsewhere [28]. Low-temperature data were collected using dynamic resonance system (DRS) 'modulus II' electronics and an Orange helium-flow cryostat, as described by McKnight et al. [29]. The sample was held across a pair of faces directly between the transducers. The automated sequence involved collection of spectra at $30 \mathrm{~K}$ intervals during cooling from $\sim 305$ to $\sim 5 \mathrm{~K}$, with a period of $20 \mathrm{~min}$ allowed for thermal equilibration at each temperature. This was followed by heating between $\sim 5$ and $\sim 300 \mathrm{~K}$, with data collection at $5 \mathrm{~K}$ intervals and the same thermal equilibration period at each temperature. Each spectrum contained 65000 data points in the frequency range $100-1200 \mathrm{kHz}$. Measured temperatures are believed to be accurate to within $\pm 1 \mathrm{~K}$, and temperature stability during data collection is better than $\pm 0.1 \mathrm{~K}$.

High-temperature spectra were collected with the sample balanced across a pair of corners between the tips of two alumina rods protruding into a horizontal tube furnace. In this system, the transducers are on the ends of the rods, outside the furnace, as described by McKnight et al. [30], and spectra are collected using Stanford electronics [31]. Temperature is monitored by a thermocouple sited within a few millimeters of the sample and checked from time to time against the $\alpha-\beta$ transition temperature of quartz, giving an experimental uncertainty of $\pm \sim 1 \mathrm{~K}$. Spectra were collected in heating and cooling sequences, from $\sim 300$ to $\sim 560 \mathrm{~K}$ with $\sim 20$ $\mathrm{K}$ steps, from $\sim 560$ to $\sim 650 \mathrm{~K}$ with $2 \mathrm{~K}$ steps, from $\sim 650$ to $\sim 750 \mathrm{~K}$ with $5 \mathrm{~K}$ steps, and then from $\sim 750$ to $\sim 650 \mathrm{~K}$ with $5 \mathrm{~K}$ steps, from $\sim 650$ to $\sim 560 \mathrm{~K}$ with $2 \mathrm{~K}$ steps, from $\sim 560$ to $\sim 300 \mathrm{~K}$ with $\sim 10 \mathrm{~K}$ steps. A period of $20 \mathrm{~min}$ was again allowed for thermal equilibration at each temperature. Individual spectra contained 65000 data points in the frequency range $50-1200 \mathrm{kHz}$.

\section{RESULTS}

Segments of the primary RUS spectra collected during cooling through the phase transition (Fig. 1) show sharp resonance peaks all stiffening (increasing in frequency, $f$ ) slightly with decreasing temperature from $\sim 760 \mathrm{~K}$ down to $\sim 700 \mathrm{~K}$, and then softening steeply to a minimum at $\sim 620 \mathrm{~K}$, which is taken to be the transition point. The softening trend then reverses and stiffening occurs down to the lowest temperature reached $(7 \mathrm{~K})$. The width at half maximum height, $\Delta f$, of all the peaks peaks broaden immediately below the transition point (Fig. 1a) and there is an additional interval of peak broadening below $200 \mathrm{~K}$ (Fig. 1b).
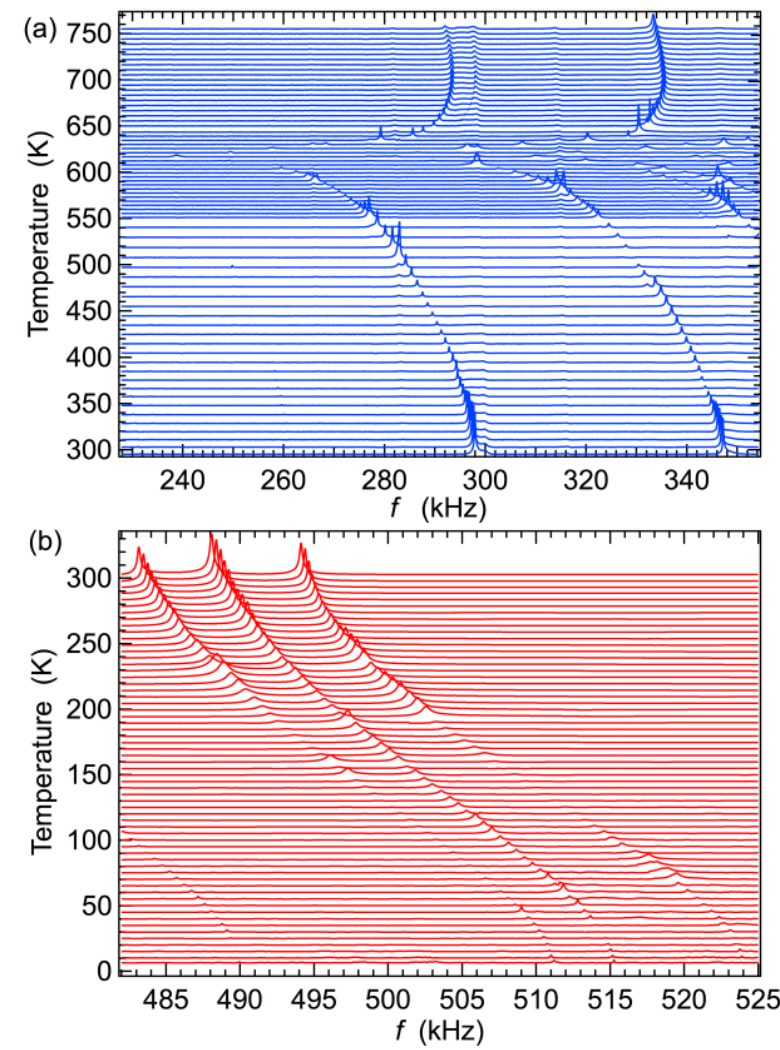

FIG. 1 (colour online). Segments of RUS spectra collected from a rectangular parallelepiped of $\mathrm{GeTe}$ with dimensions $1.977 \times 3.283 \times 4.611 \mathrm{~mm}^{3}$ and mass $0.1822 \mathrm{gm}$. Each spectrum has been offset up the y-axis in proportion to the temperature at which it was collected. (a) Cooling sequence, $\sim 760 \mathrm{~K}$ to room temperature. (b) Heating sequence, $\sim 7 \mathrm{~K}$ to room temperature.

Fitting of selected peaks provides a quantitative picture of the qualitative patterns shown by the primary spectra (Fig. 2). Variations of $f^{2}$ give the temperature dependence of predominantly shear elastic constants, and $Q^{-1}(=\Delta f / f)$ is a measure of acoustic loss. The total amount of softening with respect to the parent cubic structure is $\sim 45 \%$ at the transition point but reduces to a more nearly constant value of $\sim 30 \%$ at lower 
temperatures (Fig. 2a). This form of non-linear recovery below $T_{\text {tr }}(\sim 620 \mathrm{~K}$ on cooling and $\sim 630 \mathrm{~K}$ on heating), is closely similar to that expected for tricritical evolution of the order parameter (e.g. Refs 27, 32).

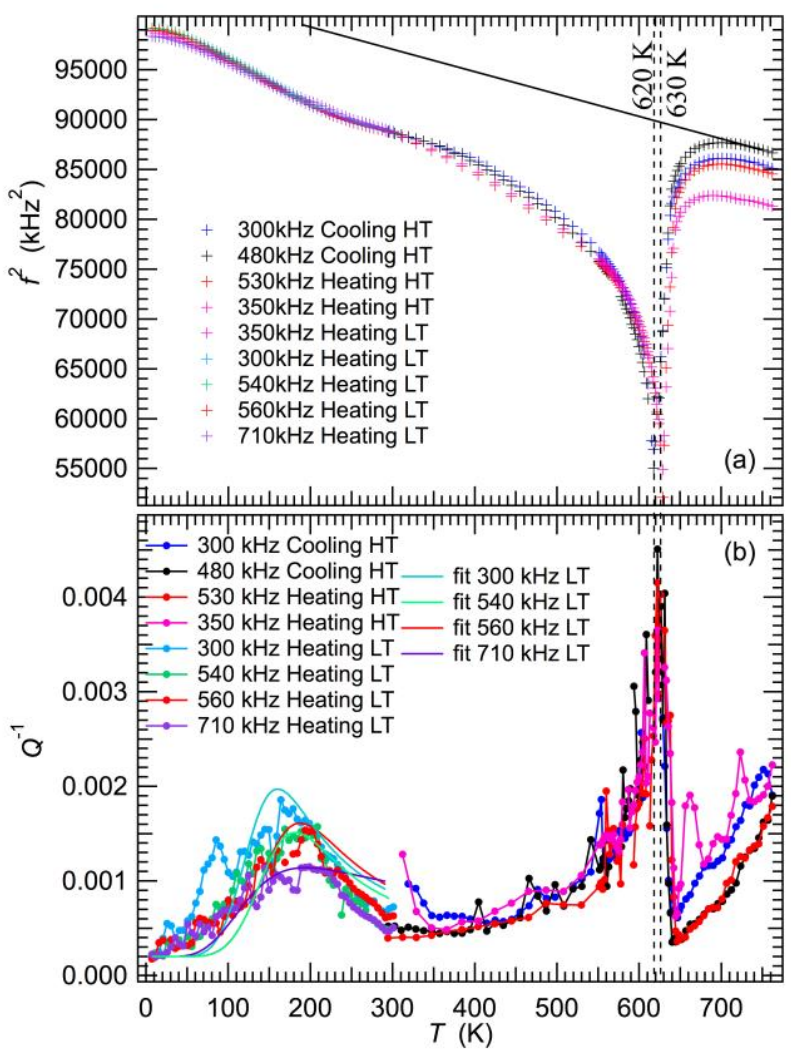

FIG. 2 (colour online). (a) Temperature-dependence of $f^{2}$ for selected resonances, scaled to overlap near room temperature. A small hysteresis of the transition point between heating $(\sim 630 \mathrm{~K})$ and cooling $(\sim 620 \mathrm{~K})$ indicates weak first order character. The straight line is a fit to data collected at the highest temperatures and extrapolated to lower temperatures in order to represent reference values, $f_{\mathrm{o}}^{2}$, of the cubic structure. (b) Temperature-dependence of $Q^{-1}$ from selected resonances, showing a marked break in trend at $\sim 650 \mathrm{~K}$, a peak in the vicinity of the minimum in $f^{2}$, and frequency-dependent maxima near $180 \mathrm{~K}$.

\section{DISCUSSION}

\section{A. Order/disorder component}

From Landau theory, the excess entropy for a phase transition is given by $-a / 2$, where $a$ is the coefficient for the second order term in the order parameter, $q$. The change in heat capacity at $T=T_{\mathrm{c}}, \Delta C_{\mathrm{p}}, \mathrm{Tc}_{\mathrm{c}}$, of the second order transition in SnTe is $\sim 0.5 \mathrm{~J} . \mathrm{mole}^{-1} . \mathrm{K}^{-1}$ [33]. For an excess free energy given by $\frac{1}{2} a\left(T T_{c}\right) q^{2}+\frac{1}{4} b q^{4}$, the excess heat capacity varies as $a T / 2 T_{\text {c }}$ [34], so that $a=$ $2 \Delta C_{\mathrm{p}, \mathrm{Tc}}=\sim 1 \mathrm{~J} \cdot \mathrm{mole}^{-1} \cdot \mathrm{K}^{-1}$. The total excess entropy for the change from $q=0$ to $q=1$ is then $\sim-0.5$ $\mathrm{J} . \mathrm{mole}^{-1} \cdot \mathrm{K}^{-1}$, which would be typical of a displacive transition mechanism. At small values of $x$ in $\mathrm{Sn}_{x} \mathrm{Ge}_{1-x} \mathrm{Te}$, the transition remains second order [20,33,35] and a classic step-like softening of elastic constants is observed, fitting with expectations for the displacive limit to which Landau theory refers [36-38]. $\Delta C_{\mathrm{p}}$ increases with increasing Ge content [35], however, implying that the excess entropy also increases, as would be expected if there is an increasing configurational contribution. Estimates of the magnitude of the excess entropy from integration of the excess heat capacity for GeTe samples with a range of stoichiometries are in the range $0.9-3.1 \mathrm{~J} \cdot \mathrm{mole}^{-1} \cdot \mathrm{K}^{-1}$ [39], though this is still less than than the expected value of $-\mathrm{R} \ln 2=-5.8 \mathrm{~J} \cdot \mathrm{mole}^{-1} \cdot \mathrm{K}^{-1}$ for the simplest $\mathrm{AB}$ ordering process.

Following Slonczewski and Thomas [40], the magnitude of softening at a second order transition with strain, $e$, coupled as $\lambda e q^{2}$ scales approximately with $\lambda^{2} / b$, where the value of the $b$ coefficient is approximately $a T_{\mathrm{c}}$. Antiferromagnetic ordering in $\mathrm{CoF}_{2}$ below $\sim 39 \mathrm{~K}$ has an excess entropy close to $-\mathrm{R} \ln 2$ and is accompanied by spontaneous strains of $\sim 0.001$ and $\sim 3 \%$ softening of the shear modulus [41]. To first approximation, allowing for the same configurational entropy, an order of magnitude increase in $\lambda$ and an order of magnitude increase in $T_{\mathrm{c}}$ should lead to an order of magnitude increase in the amount of softening in GeTe, which is essentially what is observed. In other words, the elastic softening is permissive of a high excess entropy, consistent with a significant configurational component. Moreover, the simplest model for order/disorder gives an evolution of the order parameter which is not so far from tricritical in form [41].

\section{B. Acoustic loss}

$Q^{-1}$ values at first reduce with falling temperature but have a sharp increase below $\sim 644 \mathrm{~K}$ on heating and $\sim 640 \mathrm{~K}$ on cooling towards maxima which, within experimental uncertainty, coincide with the minima in $f^{2}$ (Fig. 2b). Variations of $Q^{-1}$ below $T_{\text {tr }}$ also display typical aspects of the patterns of anelastic loss seen at improper ferroelastic transitions in perovskites. The peak at $T_{\text {tr }}$ closely resembles the peak seen at the transition temperature for octahedral tilting in $\mathrm{EuTiO}_{3}[42,43]$, $\mathrm{SrZrO}_{3}$ [27] and $\mathrm{Ca}_{0.2} \mathrm{Sr}_{0.8} \mathrm{TiO}_{3}$ [44]. The plateau of high $Q^{-1}$ between $\sim 500$ and $\sim 300 \mathrm{~K}$ results from the motion under external stress of ferroelastic twin walls in an effectively viscous medium. Finally, the frequency-dependent Debye peak at $\sim 180 \mathrm{~K}$ is typical of the effects of pinning of the twin walls by defects through some freezing interval, as seen for the tilting transition in $\mathrm{KMnF}_{3}$ [45] and for the ferroelectric transition in $\mathrm{Pb}\left(\mathrm{In}_{1 / 2} \mathrm{Nb}_{1 / 2}\right) \mathrm{O}_{3}-\mathrm{Pb}\left(\mathrm{Mg}_{1 / 3} \mathrm{Nb}_{2 / 3}\right) \mathrm{O}_{3}-\mathrm{PbTiO}_{3}$. [46].

Similar patterns of acoustic loss are seen at lower frequencies in $\mathrm{LaAlO}_{3}$ and $(\mathrm{Ca}, \mathrm{Sr}) \mathrm{TiO}_{3}$ where the overall behavior is due to forward and back motion of the needle tips of ferroelastic twin walls and the freezing mechanism is essentially pinning of the twin walls by oxygen vacancies [47-50]. Under the low stress and higher frequency conditions of an RUS experiment, the twin wall displacement mechanism most likely involves lateral motions of ledges along the length of the walls [51-53]. For present purposes, values of the temperatures, $T_{\mathrm{m}}$, at which the Debye loss peaks in Figure $2 \mathrm{~b}$ have a maximum, $Q_{\mathrm{m}}{ }^{1}$, were first determined by fitting a polynomial function to data between $\sim 10 \mathrm{~K}$ and $200 \mathrm{~K}$. A thermally activated loss mechanism with relaxation time, $\tau$, given by the condition $\omega \tau=1$ (angular frequency $\omega=2 \pi f$ ) at $T_{\mathrm{m}}$ is expected to follow $={ }_{0} \exp \left(U / \mathrm{k}_{\mathrm{B}} T\right) ; U$ is the activation energy, $\tau_{\mathrm{o}}$ the reciprocal of the attempt frequency and $k_{B}$ the Boltzmann constant. An Arrhenius plot gives $U / \mathrm{k}_{\mathrm{B}}=$ $763 \pm 53 \mathrm{~K}(U=0.066 \pm 0.005 \mathrm{eV})$ and $\tau_{\mathrm{o}}=3 \pm 1 \times 10^{-8}$ 
s (Fig. 3). This value of $U$ is close to the activation energy barrier of $0.11 \mathrm{eV}$ obtained by DFT calculation for a switching process that involves swapping of shorter and longer $\mathrm{Ge}-\mathrm{Te}$ bonds in which the Ge atoms move by $\sim 0.3 \AA$ [24]. The experimental result therefore appears to confirm the suggestion in Ref. [24] that such movements could be important in the mechanism of domain wall movement. Mechanisms involving vacancies can probably be ruled out on the basis that activation energies for diffusion of $\mathrm{Ge}$ in $\mathrm{GeTe}$ are expected to have values $\geq \sim 1 \mathrm{eV}$ [54].

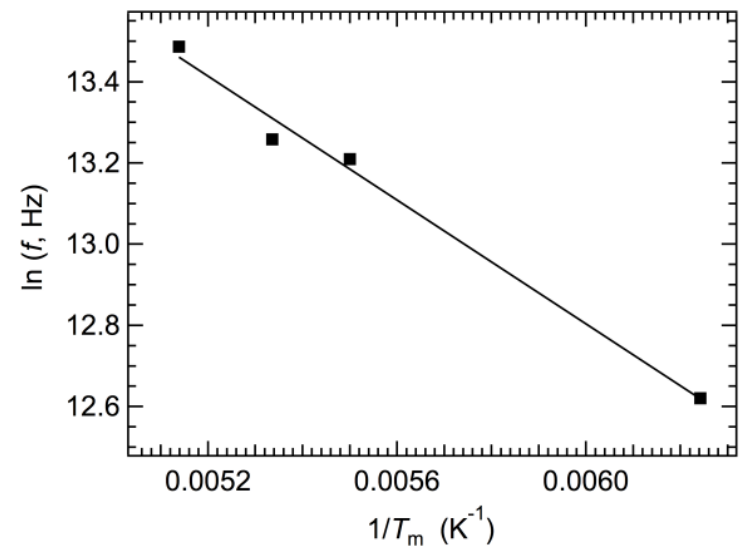

FIG. 3. Arrhenius plot of frequency, $f$, versus $1 / T_{\mathrm{m}}$, from the data of Table I. An activation energy $U=0.066 \pm 0.005 \mathrm{eV}$ is obtained from the slope and $\tau_{\mathrm{o}}=2.8 \times 10^{-8} \pm \sim 1 \times 10^{-8} \mathrm{~s}$ from one over the intercept.

Each Debye loss peak can also be fit using the expression $[45,53,55,56]$

$$
Q^{1}(T)=Q_{\mathrm{m}}{ }^{1} \cosh \frac{U}{\mathrm{k}_{\mathrm{B}} r_{2}()} \frac{1}{T} \frac{1}{T_{\mathrm{m}}} \div \quad \text { }
$$

where $r_{2}(\beta)$ relates to the width of a Gaussian spread of relaxation times. The parameter $\beta$ is a measure of the width of the Gaussian distribution, as illustrated in Figure 4-6 of Nowick and Berry [25], and is zero for a standard anelastic solid. Fits of this expression to the data have been added to Figure $2 \mathrm{a}$ and give values of $U / k_{\mathrm{B}} r_{2}(\beta)$ in the range $\sim 350-650 \mathrm{~K}$. If $U=0.066 \mathrm{eV}$ is assumed from the simpler treatment, the fits give $r_{2}(\beta)$ in the range $1.2-2.2$, with the value near $\sim 2$ being poorly constrained (Table I). The implication is that there is a single pinning mechanism with only a small spread of relaxation times $\left(\beta \sim 0, r_{2}(\beta) \sim 1\right)$.

Table I. Fit parameters for Debye-like loss peaks.

\begin{tabular}{lllll}
\hline \hline $\begin{array}{l}\text { Frequency } \\
\text { at } T_{\mathrm{m}}(\mathrm{Hz})\end{array}$ & $T_{\mathrm{m}}(\mathrm{K})$ & $Q_{\mathrm{m}}^{-1}$ & $r_{2}(\beta)$ & $U(\mathrm{eV})$ \\
\hline 302640 & 160.2 & 0.00177 & $1.4 \pm 0.1$ & 0.066 \\
545230 & 181.8 & 0.00131 & $1.2 \pm 0.1$ & 0.066 \\
572820 & 187.4 & 0.00141 & $1.2 \pm 0.2$ & 0.066 \\
719710 & 194.6 & 0.00094 & $2.2 \pm 0.3$ & 0.066 \\
\hline \hline
\end{tabular}

\section{Precursor softening dynamics}

Precursor softening of the elastic constants as an improper ferroelastic transition point is approached from above provides insights into dynamic effects because linear/quadratic coupling between the non-zero strains and the driving order parameter does not contribute any softening when $q=0$. Fluctuations relating to dispersion of the soft mode can be responsible and would be expected to conform to a phenomenological description of the form $C_{i k}=A_{i k}\left(\begin{array}{ll}T & T_{\mathrm{c}}\end{array}\right)$, where $C_{i k}$ is the amount of softening of single crystal elastic constants and $A_{i k}$ is a material constant. The value of the exponent $\kappa$, between $1 / 2$ and 2, depends on the anisotropy and dispersion of soft branches round the critical point of the soft mode [32,57-60].

Alternatively, the dynamical effects ahead of a ferroelectric transition can be due to the development of polar nanoregions. These are purely dynamic immediately below the Burns temperature but can become quasi-static at a temperature $T^{*}$ before they freeze. Softening of the shear modulus occurring in this way in $\mathrm{Pb}\left(\mathrm{Mg}_{1 / 3} \mathrm{Nb}_{2 / 3}\right) \mathrm{O}_{3}$ [61] and $\mathrm{Pb}\left(\mathrm{Sc}_{0.5} \mathrm{Ta}_{0.5}\right) \mathrm{O}_{3}$ [62] can be represented by a Vogel-Fulcher expression $C_{i k}=A_{i k} \exp U / k_{\mathrm{B}}\left(\begin{array}{ll}T & T_{\mathrm{VF}}\end{array}\right)$, where $T_{\mathrm{VF}}$ is the zero-frequency freezing temperature. The extent of precursor softening for GeTe is expressed here as $\Delta f^{2}=$ $\left(f_{\mathrm{o}}^{2}-f^{2}\right)$, where $f_{\mathrm{o}}^{2}$ is the square of the resonance frequency obtained from a linear fit to the highest temperature data, extrapolated down to $T_{\text {tr }}$ (Fig. 2a). $\Delta f^{2}$ for resonances with frequency near $530 \mathrm{kHz}$ (heating sequence) or $480 \mathrm{kHz}$ (cooling sequence) at room temperature are shown in Figure 4. The power law description, with a value of $T_{\mathrm{c}}=581 \mathrm{~K}$ taken from the strain analysis of data from Ref. [15], would not provide a good description (Fig. 4a) but the Vogel-Fulcher expression with $U=0.10 \pm 0.06 \mathrm{eV}$ and $T_{\mathrm{VF}}=520 \pm 30$ or $T_{\mathrm{VF}}=530 \pm 30 \mathrm{~K}$ can for heating or cooling respectively (Fig. 4b). Remarkably, the activation energy for the fits is almost the same as for domain wall freezing, which appears to imply that the energy barrier associated with the freezing process is also determined by changing the configuration of short and long $\mathrm{Ge}-\mathrm{Te}$ bonds. 

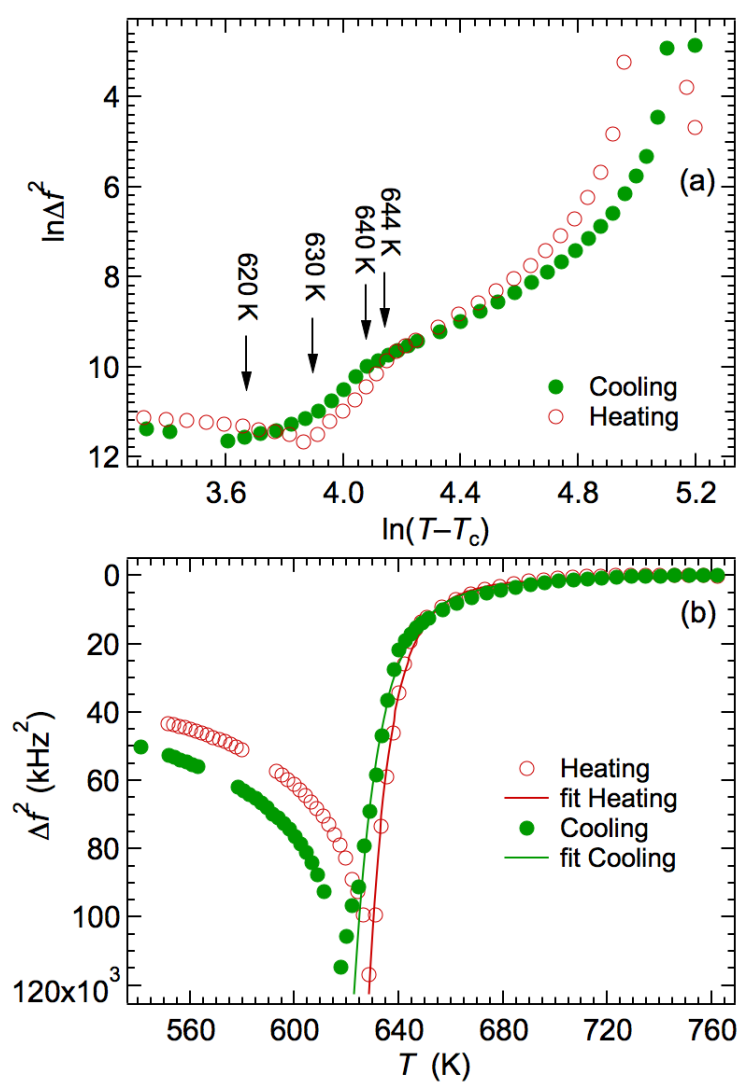

FIG. 4 (colour online). Variations of $\Delta f^{2}=\left(f_{\mathrm{o}}^{2}-f^{2}\right)$ with temperature. (a) The data above the transition temperature do not have a linear temperature dependence with slope between $-1 / 2$ and -2 , as would be expected for a power law description for $T_{\mathrm{c}}=581 \mathrm{~K}$. (b) Curves through the data above $T_{\text {tr }}$ are fits of a Vogel-Fulcher equation.

We propose that the precursor elastic softening seen in all mechanical resonances of the GeTe sample reflects coupling of the acoustic modes with a central relaxational mode due to dynamical clustering of polar regions in the manner reported recently for the ferroelectric transition in $\mathrm{Pb}\left(\mathrm{Fe}_{0.5} \mathrm{Nb}_{0.5}\right) \mathrm{O}_{3}$ [63]. The central mode would involve flipping of the polarization or displacements of boundaries between ordered clusters, and $T_{\mathrm{VF}}$ represents the temperature at which this motion would be expected to cease if the ferroelectric transition did not intervene. A test would be observation of a mode (or modes) with relaxation times perhaps in the vicinity of $\sim 10^{-10} \mathrm{~s}$, as appears to have been detected in terahertz spectra by Kadlec et al. [64]. Such a central mode would also be expected to couple with optic modes which might therefore provide indirect evidence of its unseen presence. There is a distinct kink in the $\Delta f^{2}$ data at $\sim 640-645 \mathrm{~K}$ during both heating and cooling, (Fig. 4a) and this is interpreted as representing the temperature, $T^{*}$, where the clusters acquire some static component. The change in trend of $\Delta f^{2}$ coincides with the abrupt change in trend for $Q^{-1}$ (Fig. 2b), as would be expected if it marks the development of some ferroelastic microstructure with falling temperature - for which the most likely form would be tweed. Some degree of strain coupling of the dynamical clustering at $T>T^{*}$ is evidenced by the persistence of relatively high values of $Q^{-1}$ up to the highest temperatures of the measurements presented here.

The acoustic data complement the evidence of clustering from pair distribution analysis of diffraction data presented in Ref. [15]. The sample used in the present study is from the same original boule and close agreement for the transition temperature implies that the compositions are closely similar. Hudspeth et al. [15] described ordered clusters with dimension $\sim 20 \AA$ in the stability field of the cubic structure, with a steep increase in correlation length between $650 \mathrm{~K}$ and $500 \mathrm{~K}$. This interval coincides almost exactly with the interval over which $Q^{-1}$ shows a peak through the transition. Correlations of the distorted GeTe polyhedra can therefore be understood as being at first dynamic on the cluster length scale and then quasi-static below $\sim 645 \mathrm{~K}$ when the length scale of the correlations starts to increase. As pointed out already by Chatterji et al. [16], the positive volume strain observed for GeTe has essentially the same form as seen in $\mathrm{LaMnO}_{3}$ where the transition mechanism involves ordering of $\mathrm{MnO}_{6}$ octahedra with Jahn-Teller distortions.

\section{CONCLUSIONS}

Elastic and anelastic anomalies provide a sensitive window on the strength and dynamics of strain coupling effects which accompany structural, magnetic and electronic phase transitions in perovskites [53]. In the case of GeTe, large softening of the shear elastic constants and strong coupling of the driving order parameter(s) with shear strain are consistent with improper ferroelastic character for the $F m 3 m-R 3 m$ transition. The temperature dependence of the strain evolution and the form of the elastic softening is consistent with a mean field description of a displacive transition which is close to tricritical. The magnitude of the softening, however, is permissive of a significant configurational component coming from order/disorder of distorted GeTe polyhedra. The softening data also provide evidence for the influence of an unseen central relaxational mode. An activation energy barrier of $\sim 0.1$ $\mathrm{eV}$ seems to control both the dynamics of the ordering process and of the resulting ferroelastic microstructures. As such it is likely to represent the thermal barrier for switching processes.

GeTe already has remarkable macroscopic properties but the existence of polar domains, tweed microstructures and possible combinations of $180^{\circ}$ and $71 / 109^{\circ}$ twin walls opens up additional possibilities in the context of domain engineering and the use of transformation microstructures for providing device properties $[65,66]$. For example, the proximity to a metal - insulator transition [5], due to the particular coupling between atomic structure and electronic structure, means that is inevitable that the electrical properties of twin walls will differ from those of the domains.

\section{ACKNOWLEDGMENTS}

RUS facilities have been established and maintained in Cambridge through grants from the Natural Environment Research Council and the Engineering and Physical Sciences Research Council of Great Britain to MAC (NE/B505738/1, NE/F17081/1, EP/I036079/1). Dexin Yang thanks the China Scholarship Council. 


\section{APPENDIX: SYMMETRY AND STRAIN \\ ANALYSIS}

Ferroelectric dipoles typically develop in GeTe due to displacements of $\mathrm{Ge}$ and $\mathrm{Te}$ atoms following the evolution of order parameter components that belong to the irreducible representation $\Gamma_{4}^{-}$of parent space group $F m \overline{3} \mathrm{~m}$. As well as being ferroelectric at room temperature, GeTe is in principle improper ferroelastic due to coupling of the ${ }_{4}$ order parameter with strain. Following Rehwald and Lang [36] and Sugai et al. [21], the Landau expansion for the excess free energy, $G$, including saturation is

$$
\begin{aligned}
G & =\frac{1}{2} a_{\mathrm{s}} \text { coth } \frac{\mathrm{s}}{T} \div \operatorname{coth} \frac{\mathrm{s}}{T_{\mathrm{c}}} \div\left(q_{1}^{2}+q_{2}^{2}+q_{3}^{2}\right) \\
& +\frac{1}{4} b\left(q_{1}^{2}+q_{2}^{2}+q_{3}^{2}\right)^{2}+\frac{1}{4} b\left(q_{1}^{4}+q_{2}^{4}+q_{3}^{4}\right) \\
& +\frac{1}{6} c\left(q_{1}^{2}+q_{2}^{2}+q_{3}^{2}\right)^{3} \\
& +\frac{1}{6} c\left(q_{1}^{2}+q_{2}^{2}+q_{3}^{2}\right)\left(q_{1}^{2} q_{2}^{2}+q_{1}^{2} q_{3}^{2}+q_{2}^{2} q_{3}^{2}\right) \\
& +\frac{1}{6} c\left(q_{1}^{6}+q_{2}^{6}+q_{3}^{6}\right)+{ }_{1} e_{\mathrm{a}}\left(q_{1}^{2}+q_{2}^{2}+q_{3}^{2}\right) \\
& +{ }_{2} \sqrt{3} e_{\mathrm{o}}\left(q_{1}^{2} \quad q_{2}^{2}\right) e_{\mathrm{t}}\left(q_{1}^{2}+q_{2}^{2} 2 q_{3}^{2}\right) \\
& +{ }_{3}\left(e_{4} q_{2} q_{3}+e_{5} q_{1} q_{3}+e_{6} q_{1} q_{2}\right) \\
& +\frac{1}{4}\left(C_{11}^{\mathrm{o}} C_{12}^{\mathrm{o}}\right)\left(e_{\mathrm{o}}^{2}+e_{\mathrm{t}}^{2}\right)+\frac{1}{6}\left(C_{11}^{\mathrm{o}}+2 C_{12}^{\mathrm{o}}\right) e_{\mathrm{a}}^{2} \\
& +\frac{1}{2} C_{44}^{\mathrm{o}}\left(e_{4}^{2}+e_{5}^{2}+e_{6}^{2}\right) .
\end{aligned}
$$

$q_{1}-q_{3}$ are order parameter components, $a, b, c$, etc., are normal Landau coefficients, $\Theta_{s}$ is the saturation temperature for the order parameter, $T_{\mathrm{c}}$ is the critical temperature, $\lambda_{1}, \lambda_{2}, \lambda_{3}$ are coupling coefficients, $C_{11}^{\mathrm{o}}$, $C_{12}^{\mathrm{o}}, C_{44}^{\mathrm{o}}$, are bare elastic constants, $e_{4}, e_{5}, e_{6}$ are strain components. The symmetry-adapted strains, $e_{\mathrm{a}}, e_{\mathrm{o}}$ and $e_{\mathrm{t}}$ are combinations of the linear strain components $e_{1}, e_{2}$, and $e_{3}$, as

$$
\begin{aligned}
& e_{\mathrm{a}}=\left(e_{1}+e_{2}+e_{3}\right) \\
& e_{\mathrm{o}}=\left(\begin{array}{ll}
e_{1} & e_{2}
\end{array}\right) \\
& e_{t}=\frac{1}{\sqrt{3}}\left(\begin{array}{lll}
2 e_{3} & e_{1} & e_{2}
\end{array}\right)
\end{aligned}
$$

Space groups, non-zero order parameter components and lattice vectors for the symmetry subgroups of $F m 3 \mathrm{~m}$ associated with 4 are listed in full in Table A.I. Order parameter components for the $R 3 m$ structure are $q_{1}=q_{2}=q_{3} \neq 0$, and equation (A.1) reduces to

$$
\begin{aligned}
G & =\frac{3}{2} a \text { s } \text { coth } \frac{\mathrm{s}}{T} \div \operatorname{coth} \frac{\mathrm{s}}{T_{\mathrm{c}}} \div q_{1}^{2}+\frac{9}{4} b q_{1}^{4}+\frac{3}{4} b q_{1}^{4} \\
& +\frac{27}{6} c q_{1}^{6}+\frac{9}{6} c q_{1}^{6}+\frac{3}{6} c q_{1}^{6}+3{ }_{1} e_{\mathrm{a}} q_{1}^{2}+{ }_{3} q_{1}^{2}\left(e_{4}+e_{5}+e_{6}\right) \\
& +\frac{1}{4}\left(C_{11}^{\mathrm{o}} \quad C_{12}^{\mathrm{o}}\right)\left(e_{\mathrm{o}}^{2}+e_{\mathrm{t}}^{2}\right)+\frac{1}{6}\left(C_{11}^{\mathrm{o}}+2 C_{12}^{\mathrm{o}}\right) e_{\mathrm{a}}^{2} \\
& +\frac{1}{2} C_{44}^{\mathrm{o}}\left(e_{4}^{2}+e_{5}^{2}+e_{6}^{2}\right) .
\end{aligned}
$$

Table A.I. Space groups, non-zero order parameter components, and lattice vectors (origin $=(0,0,0)$ ), for the symmetry subgroups of $\mathrm{Fm} 3 \mathrm{~m}$ associated with active representation $\quad{ }_{4}$, as obtained from the group theory program ISOTROPY [67].

\begin{tabular}{llll}
\hline $\begin{array}{l}\text { Space } \\
\text { group }\end{array}$ & $\begin{array}{l}\text { Order parameter } \\
\text { components }\end{array}$ & $\begin{array}{l}\text { Relationships between } \\
\text { order parameter } \\
\text { components }\end{array}$ & Lattice vectors \\
\hline$F m \overline{3} m$ & 000 & & $(0,0,0)(0,0,0)(0,0,0)$ \\
$I 4 m m$ & $q_{1} 00$ & & $(0,1 / 2,1 / 2)(0,-1 / 2,1 / 2)(1,0,0)$ \\
$I m m 2$ & $q_{1} q_{2} 0$ & $q_{1}=q_{2}$ & $(-1 / 2,1 / 2,0)(0,0,1)(1 / 2,1 / 2,0)$ \\
$R 3 m$ & $q_{1} q_{2} q_{3}$ & $q_{1}=q_{2}=q_{3}$ & $(-1 / 2,1 / 2,0)(0,-1 / 2,1 / 2)(1,1,1)$ \\
$C m$ & $q_{1} q_{2} 0$ & $q_{1} \neq q_{2}$ & $(-1,0,0)(0,0,1)(1 / 2,1 / 2,0)$ \\
$C m$ & $q_{1} q_{2} q_{3}$ & $q_{1}=q_{2} \neq q_{3}$ & $(1 / 2,1 / 2,1)(-1 / 2,1 / 2,0)(-1 / 2,-1 / 2,0)$ \\
$P 1$ & $q_{1} q_{2} q_{3}$ & $q_{1} \neq q_{2} \neq q_{3}$ & $(0,1 / 2,1 / 2)(1 / 2,0,1 / 2)(1 / 2,1 / 2,0)$ \\
\hline \hline
\end{tabular}

If reference axes $X, Y$ and $Z$ are chosen as being parallel to the cubic crystallographic axes, the non-zero strain components are given by

$$
e_{1}=e_{2}=e_{3}=\frac{a a_{\mathrm{o}}}{a_{\mathrm{o}}}
$$

$e_{4}=e_{5}=e_{6}=\frac{a}{a_{\mathrm{o}}} \cos \quad \approx \cos \alpha$,

where $a_{\mathrm{o}}$ is the reference parameter of the cubic structure extrapolated into the rhombohedral stability field, $a$ is the lattice parameter of the rhombohedral structure and $\alpha$ is its (pseudocubic) lattice angle. The equilibrium condition $\partial G / \partial e=0$ gives relationships 
between strains and order parameter components as

$$
e_{\mathrm{a}}=\frac{3{ }_{1} q_{1}^{2}}{\frac{1}{3}\left(C_{11}^{\mathrm{o}}+2 C_{12}^{\mathrm{o}}\right)}
$$

$e_{\mathrm{o}}=e_{\mathrm{t}}=0$

$e_{4}=e_{5}=e_{6}=\frac{{ }_{3}^{2} q_{1}^{2}}{C_{44}^{\mathrm{o}}}$.

These relationships are used here to determine the thermodynamic character of the cubic - rhombohedral transition.

Cell parameter data from single crystal neutron diffraction [17] and X-ray powder diffraction [7] are reproduced in Figure A.1a. Normally, a fit of the function [68-72]

$a_{\mathrm{o}}=a_{1}+a_{2}$ so $\operatorname{coth} \frac{\text { so }}{T} \div$
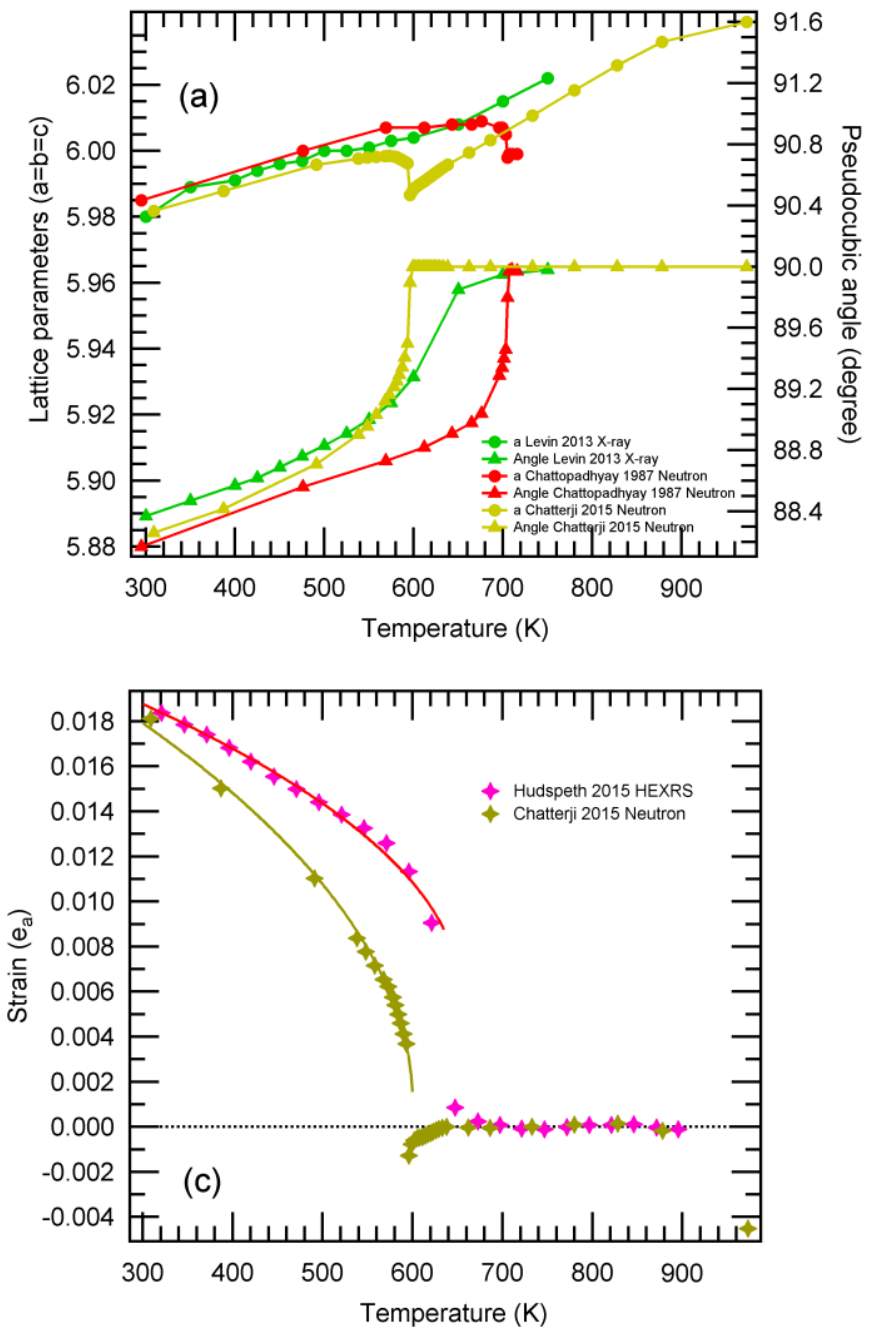

(where $\Theta_{\text {so }}$ is a saturation temperature for thermal expansion) is used to obtain the reference cubic lattice parameter, but there are insufficient data in the stability field of the cubic phase for this. However, variations of the shear strain, $e_{4}$, are to good approximation given by $\cos \alpha$ and are consistent with the known, weakly first order character of the transition (Fig. A.1b). Added to Figure A.1a and A.1b are data from the study of Chatterji et al. [16] which were not shown in the original paper, and from Hudspeth et al. [15] obtained using synchrotron $\mathrm{x}$-ray powder diffraction. The latter have been used to yield fits of Equation A.11 for $a_{0}$ and, hence, to determine the values of $e_{\mathrm{a}}$ which are given in Figure A.1c. From Equations A.8 and A.10, $e_{\mathrm{a}}$ and $e_{4}$ are expected to be linearly dependent but this appears not to be the case within reasonable experimental uncertainty (Fig. A.1d). Either there is additional higher order coupling with one or other of the strains, i.e. such as $\lambda e Q^{4}$, or one order parameter alone is not sufficient to describe the overall transformation behaviour.
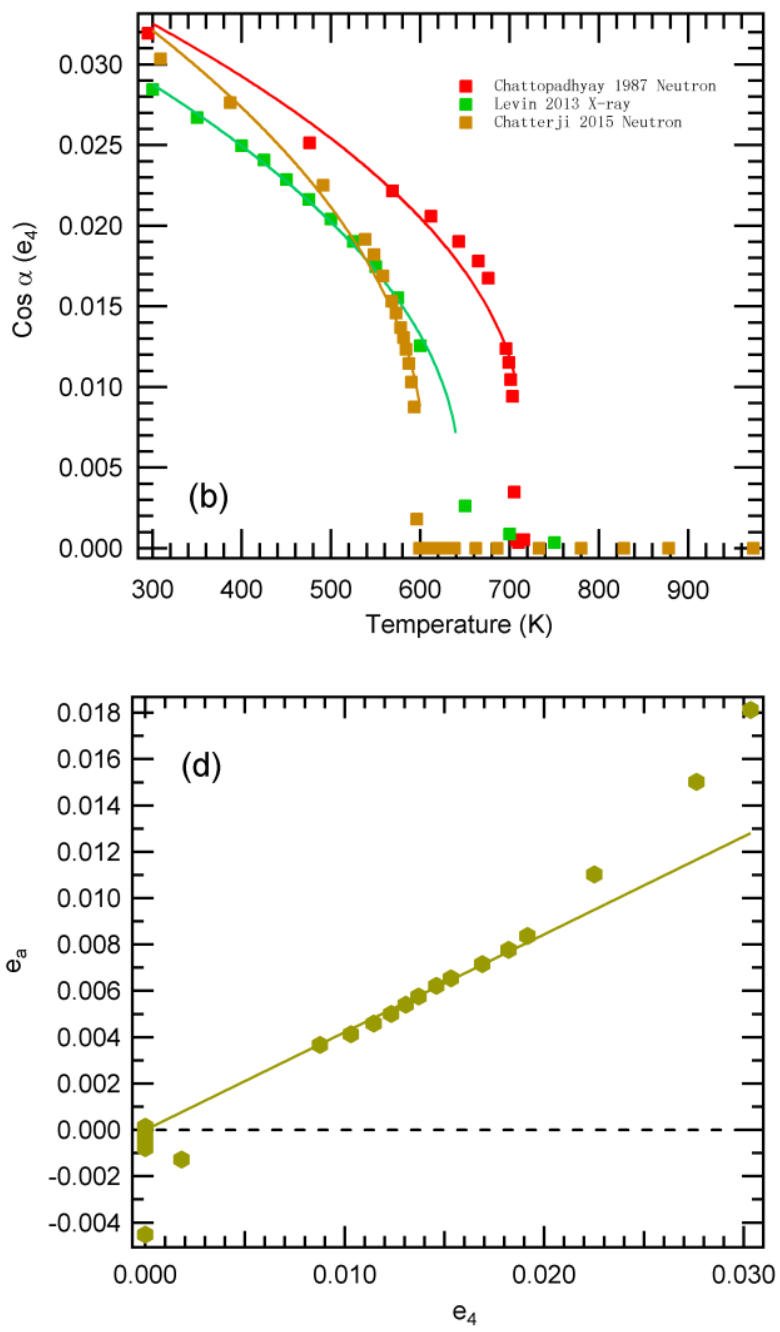

FIG. A.1. Strain analysis of the $F m \overline{3} m-R 3 m$ transition in GeTe, showing weakly first order character. (a) Cell parameter variations compiled from the literature. Straight lines fit to data points for $a$ at the highest temperatures represent the variations of $a_{0}$ extrapolated to lower temperatures. (b) Curves through the data for $\cos \alpha\left(\sim e_{4} \propto q_{1}^{2}\right)$ are fits of Equation A.12, with $T_{\text {tr }}$ fixed at values specified in the original work and values of the fit parameters given in Table A.II. (c) Curves through the data for $e_{\mathrm{a}}\left(\propto q_{1}^{2}\right)$ are fits of 
Equation A.12, with parameters given in Table A.II. (d) $e_{\mathrm{a}}$ does not scale linearly with $e_{4}$ as expected from Equations A.8 and A.10 over the entire range.

As in the case of the $\alpha-\beta$ transition in quartz [73] the evolution of the order parameter is expected to follow the standard Landau solution for a first-order transition:

$Q^{2}=\frac{2}{3} Q_{\mathrm{o}}^{2} 1+1 \frac{3}{4} \frac{T T_{\mathrm{c}}}{T_{\text {tr }} T_{\mathrm{c}}} \div \frac{1 / 2}{\div}$

where $Q_{\mathrm{o}}$ is the discontinuity in the order parameter at the transition temperature, $T_{\mathrm{tr}}$. The difference between $T_{\text {tr }}$ and the critical temperature, $T_{\mathrm{c}}$, is a measure of how far the transition is from being thermodynamically continuous and, although this should include the saturation temperature from Equation A.5, it is usually adequate to ignore it in fitting data above room temperature. Figures A.1b and A.1c show fits of a function of the form of Equation A.12 to data for $e_{4}$ and $e_{\mathrm{a}}$ derived from the lattice parameters of Levin et al. [7], Chatterji et al. [16] and Hudspeth et al. [15]. $T_{\text {tr }}$ was fixed at the experimental values and the resulting fit parameters are listed in Table A.II. Just as found for the first order transition at compositions towards the Sn-rich end of the $\mathrm{Sn}_{x} \mathrm{Ge}_{1-x} \mathrm{Te}$ solid solution [18], the Landau solution provides a good representation of the strain variations. Although the transition temperature varies between samples, the pattern of strain evolution is similar and values of $\left(T_{\mathrm{tr}}-T_{\mathrm{c}}\right)$ fall in the range $1-50 \mathrm{~K}$. Changes of $T_{\text {tr }}$ between samples of GeTe are widely attributed to the effects of changing stoichiometry (e.g. Ref. 17]), and might also contribute to changes in the strength of strain/order parameter coupling or changes in the thermodynamic character of the transition but there are insufficient data to test this possibility systematically through correlations of $\left(T_{\mathrm{tr}}-T_{\mathrm{c}}\right)$ with the value of $T_{\text {tr }}$, say.

Table A.II. Fit parameters from Figure A.1 using Equation A.12.

\begin{tabular}{lllll}
\hline \hline Source of data & $\cos \alpha_{\mathrm{o}} / e_{\mathrm{ao}}$ & $\begin{array}{l}T_{\mathrm{tr}}(\mathrm{K}) \\
(\text { fixed })\end{array}$ & $\begin{array}{l}T_{\mathrm{c}} \\
(\mathrm{K})\end{array}$ & $\begin{array}{l}T_{\mathrm{tr}}-T_{\mathrm{c}} \\
(\mathrm{K})\end{array}$ \\
\hline $\begin{array}{l}\text { Chattopadhyay } \\
\text { and Boucherle }\end{array}$ & $\begin{array}{l}\cos \alpha_{\mathrm{o}}= \\
0.0109\end{array}$ & 705 & 680 & 25 \\
$\begin{array}{l}\text { [17] } \\
\begin{array}{l}\text { Chatterji } \text { et al. } \\
\text { [16] }\end{array}\end{array}$ & $\begin{array}{l}\cos \alpha_{\mathrm{o}}= \\
0.0086\end{array}$ & 600 & 589 & 11 \\
$\begin{array}{l}\text { Levin } \text { et al. } \text { [7] } \\
\text { X-ray }\end{array}$ & $\begin{array}{l}\cos \alpha_{\mathrm{o}}= \\
0.0070\end{array}$ & 640 & 630 & 10 \\
$\begin{array}{l}\text { Chatterji } \text { et al. } \\
{[16]}\end{array}$ & $\begin{array}{l}e_{\mathrm{ao}}= \\
0.0015\end{array}$ & 600 & 599 & 1 \\
$\begin{array}{l}\text { Hudspeth } \text { et al. } \\
\text { [15] }\end{array}$ & $\begin{array}{l}e_{\mathrm{ao}}= \\
0.0088\end{array}$ & 635 & 581 & 54 \\
\hline \hline
\end{tabular}

\section{References}

[1] M. Wuttig and N. Yamada, Nat. Mater. 6. 824 (2007).

[2] G. Bruns, P. Merkelbach, C. Schlockermann, M. Salinga, M. Wuttig, T. D. Happ, J. B. Philipp and M. Kund, Appl Phys Lett 95, 043108 (2009).
[3] A.V. Kolobov, M. Krbal, P. Fons, J. Tominaga and T. Uruga, Nature Chem 3, 311 (2011).

[4] X. Zhou, W. Dong, H. Zhang and R. E. Simpson, Scientific Reports 5, 11150 (2015).

[5] P. Nukala, R. Agarwal, X. Qian, M.H. Jang, S. Dhara, K. Kumar, A. T. C. Johnson, J. Li and R. Agarwal, Nano Lett 14, 2201 (2014).

[6] G. J. Snyder and E. S. Toberer, Natur Mater 7, 105 (2008).

[7] E. M. Levin, M. F. Besser and R. Hanus, J Appl Phys 114, 083713 (2013).

[8] M. J. Polking, J. J. Urban, D. J. Milliron, H. Zheng, E. Chan, M. A. Caldwell, S. Raoux, C. F. Kisielowski, J. W. Ager III, R. Ramesh and A. P. Alivisatos, Nano Lett 11, 1147 (2011).

[9] M. Chen, K. A. Rubin and R. W. Barton, Appl Phys Lett 49, 502 (1986).

[10] A. H. Edwards, A. C. Pineda, P. A. Schultz, M. G. Martin, A. P. Thompson, H. P. Hjalmarson, and C. J. Umrigar, Phys Rev B 73, 045210 (2006).

[11] J.-P. Gaspard, A. Pellegatti, F. Marinelli and C. Bichara, Phil Mag B 77, 727 (1998).

[12] J. Y. Raty, V. Godlevsky, Ph. Ghosez, C. Bichara, J. P. Gaspard, and James R. Chelikowsky, Phys Rev Lett 85, 1950 (2000).

[13] P. Fons, A. V. Kolobov, M. Krbal, J. Tominaga, K. S. Andrikopoulos, S. N. Yannopoulos, G. A. Voyiatzis and T. Uruga, Phys Rev B 82, 155209 (2010)

[14] T. Matsunaga, P. Fons, A. V. Kolobov, J. Tominaga, and N. Yamada, Applied Physics Letters 99, 231907 (2011).

[15] J. M. Hudspeth, T. Chatterji, S. J. Billinge, and S. A Kimber, arXiv preprint arXiv:1506.08944 (2015).

[16] T. Chatterji, C. Kumar, and U. D. Wdowik, Phys Rev B 91, 054110 (2015).

[17] T. Chattopadhyay and J. Boucherle, J Phys C: Sol St Phys 20, 1431 (1987).

[18] E. Steigmeier and G. Harbeke, Sol St Commun 8, 1275 (1970).

[19] K. M. Rabe and J. D. Joannopoulos, Phys Rev B 36, 6631 (1987).

[20] R. Clarke, Phys Rev B 18, 4920 (1978).

[21] S. Sugai, K. Murase, T. Tsuchihira, H. Kawamura, J Phys Soc Jpn 47, 539 (1979).

[22] K. M. Rabe and J. D. Joannopoulos, Phys Rev B 36, 6631 (1987).

[23] U. D. Wdowik, K. Parlinski, S. Rols, and T. Chatterji, Phys Rev B 89, 224306 (2014).

[24] A. V. Kolobov, D. J. Kim, A. Giussani, P. Fons, J. Tominaga, R. Calarco and A. Gruverman, Appl Phys Lett Mater 2, 066101 (2014).

[25] A. S. Nowick and B. S. Berry, Anelastic Relaxation in Crystalline Solids. Academic Press: New York (1972).

[26] M. A. Carpenter and C. J. Howard, Acta Cryst B 65, 147 (2009).

[27] R. E. A. McKnight, C. J. Howard, and M. A. Carpenter, J Phys: Condens Matter 21, 015901 (2009).

[28] A. S. Migliori and J. L. Sarrao, Resonant 
Ultrasound Spectroscopy: Applications to Physics, Materials Measurements and Nondestructive Evaluation, Wiley: New York (1997).

[29] R. E. A. McKnight, M. A. Carpenter, T. W. Darling, A. Buckley, and P. A. Taylor, Am Mineral 92, 1665 (2007).

[30] R. E. A. McKnight, T. Moxon, A. Buckley, P. A. Taylor, T. W. Darling and M. A. Carpenter, J Phys: Condens Matter 20, 075229 (2008).

[31] A. Migliori and J. D. Maynard, Rev Sci Instrum 76, 121301 (2005).

[32] M. A. Carpenter and E. K. H. Salje, Eur J Min 10 , 693 (1998).

[33] I. Hatta and K. L. I. Kobayashi, Sol St Comm 22, 775 (1977).

[34] M. A. Carpenter, in Physical Properties and Thermodynamic Behaviour of Minerals, E. K. H. Salje (Ed.), NATO ASI Series C 225, 265 (1988).

[35] I. Hatta and W. Rehwald, J Phys C: Sol St Phys 10, 2075 (1977).

[36] W. Rehwald and G. K. Lang, J Phys C: Sol St Phys 8, 3287 (1975).

[37] T. Seddon, J.M. Farley, G.A. Saunders, Sol St Comm 17, 55 (1975).

[38] T. Seddon, S.C. Gupta, G.A. Saunders, Phys Lett A 56, 45 (1976).

[39] N. Kh. Abrikosov, M. A. Korzhuev, L. A. Petrov, O. A. Teplov, and G. K. Demenckii, Inorg Mat 19, 334 (1983).

[40] J. C. Slonczewski and H. Thomas, Phys Rev B 1, 3599 (1970).

[41] R. I. Thomson, T. Chatterji and M. A. Carpenter, J Phys: Condens Matter 26, 146001 (2014).

[42] L. J. Spalek, S. S. Saxena, C. Panagopoulos, T. Katsufuji, J. A. Schiemer, and M. A. Carpenter, Phys Rev B 90, 054119 (2014).

[43] J. Schiemer, L. J. Spalek, S. S. Saxena, C. Panagopoulos, T. Katsufuji and M. A. Carpenter, Europhys Lett 109, 57004 (2014).

[44] N. J. Perks, Z. Zhang, R. J. Harrison and M. A. Carpenter, J Phys: Condens Matter 26, 505402 (2014).

[45] M. Carpenter, E. Salje, and C. J. Howard, Phys Rev B 85, 224430 (2012).

[46] G. F. Nataf, Q. Li, Y. Liu, R. L. Withers, S. L. Driver, and M. A. Carpenter, J Appl Phys 113, 124102 (2013).

[47] R. J. Harrison, S. A. Redfern, and J. Street, Am Mineral 88, 574 (2003).

[48] R. J. Harrison and S. A. T. Redfern, Phys Earth Planet Int 134, 253 (2002).

[49] R. J. Harrison, S. A. T. Redfern, and E. K. H. Salje, Phys Rev B 69, 144101 (2004).

[50] M. Daraktchiev, R.J. Harrison, E.H. Mountstevens, S.A.T. Redfern, Mat Sci Eng A 442, 199 (2006).

[51] M. A. Carpenter and Z. Zhang, Geophys J Int 186,
279 (2011).

[52] E. K. H. Salje, X. Ding, Z. Zhao, T. Lookman, and A. Saxena, Phys Rev B 83, 104109 (2011).

[53] M. A. Carpenter, J Phys: Cond Matter 27, 263201 (2015).

[54] V. L. Deringer, M. Lumeii, R. P. Stoffel, and R. Dronskowski, Chem Mater 25, 2220 (2013).

[55] J. A. Schiemer, R. L. Withers, Y. Liu, and M. A. Carpenter, Chem Mat 25, 4436 (2013).

[56] M. A. Carpenter, C. J. Howard, R. E. McKnight, A. Migliori, J. B. Betts, and V. R. Fanelli, Phys Rev B 82, 134123 (2010).

[57] J. D. Axe and G. Shirane, Phys Rev B 1, 342 (1970).

[58] E. Pytte, Phys Rev B 1, 924 (1970).

[59] E. Pytte 1971 in "Structural phase transitions and soft modes", eds E. J. Samuelsen, J. Anderson, and J. Feder, NATO ASI Norway. Scandinavian University Books, Oslo, 151 (1971).

[60] U. T. Höchli, Phys Rev B 6, 1814 (1972).

[61] M. A. Carpenter, J. F. J. Bryson, G. Catalan, S. J. Zhang, and N. J. Donnelly, J Phys: Condens Matter 24, 045902 (2012).

[62] O. Aktas, E. K. H. Salje, S. Crossley, G. I. Lampronti, R. W. Whatmore, N. D. Mathur, and M. A. Carpenter, Phys Rev B 88, 174112 (2013).

[63] R. Mackeviciute, V. Goian, S. Greicius, R. Grigalaitis, D. Nuzhnyy, J. Holc, J. Banys and S. Kamba, J Appl Phys 117, 084101 (2015).

[64] F. Kadlec, C. Kadlec, P. Kužel, and J. Petzelt, Phys Rev B 84, 205209 (2011).

[65] E. K. H. Salje, ChemPhysChem 11, 940 (2010).

[66] D. D. Viehland and E. K. H. Salje, Adv Phys 63, 267 (2014).

[67] H. T. Stokes, D. M. Hatch, and B. J. Campbell, ISOTROPY (http://stokes.byu.edu/isotropy.html) (2005).

[68] M. A. Carpenter, J. Bryson, G. Catalan, and C. Howard, J Phys: Condens Matter 24, 045901 (2012).

[69] E. Salje, B. Wruck, and H. Thomas, Zeit Phys B 82, 399 (1991).

[70] H.-W. Meyer, M. A. Carpenter, A. Graeme-Barber, P. Sondergeld, and W. Schranz, Eur J Min 12, 1139 (2000).

[71] H.-W. Meyer, S. Marion, P. Sondergeld, M. A. Carpenter, K. S. Knight, S. A. T. Redfern, and M. T. Dove, Am Mineral 86, 566 (2001).

[72] P. Sondergeld, W. Schranz, A. V. Kityk, M. A. Carpenter, and E. Libowitzky, Phase Trans 71, 189 (2000).

[73] M. A. Carpenter, E. K. H. Salje, A. Graeme-Barber, B. Wruck, M. T. Dove, and K. S. Knight, Am Mineral 83, 2 (1998). 